\title{
Special Issue Dedicated to Dr. Kazuhiro Ikenaka
}

\author{
Hiroko Baba ${ }^{1} \cdot$ Yukio Yoneda $^{2}$
}

Published online: 21 November 2017

(c) Springer Science+Business Media, LLC, part of Springer Nature 2017

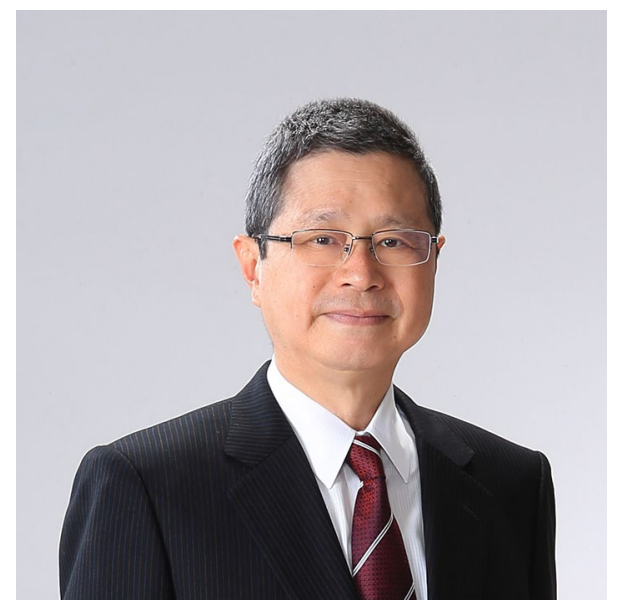

It is our great pleasure to serve as the guest editors of the Special Issue of Neurochemical Research dedicated to Dr. Kazuhiro Ikenaka.

About 30 years ago when Dr. Kazuhiro Ikenaka, also known as Kaz within the myelin research group, changed his research field from the development of anticancer agents, based on his specialty in organic chemistry, to the field of neuroscience, glial research was not yet popular in Japan. Kaz graduated from the Department of Chemistry, Faculty of Science, Osaka University in 1975. During the course of his PhD training (1975-1980) at the Institute for Protein Research, Osaka University (under Prof. Setsuro Fujii), he

Hiroko Baba

hirobaba@toyaku.ac.jp

Yukio Yoneda

yyoneda@p.kanazawa-u.ac.jp

1 Department of Molecular Neurobiology, School of Pharmacy, Tokyo University of Pharmacy and Life Sciences, 1432-1 Horinouchi, Hachioji, Tokyo 192-0392, Japan

2 Section of Prophylactic Pharmacology, Kanazawa University, Venture Business Laboratory 402, Kakuma-machi, Kanazawa, Ishikawa 920-1192, Japan

studied pyrimidine metabolism and focused on anticancer research. When he returned from his 2-year postdoctoral training (1983-1985) at the State University of New York at Stony Brook (under Prof. Masayori Inoue), Dr. Katsuhiko Mikoshiba had just become the new lab chief of the Institute for Protein Research where Kaz was an instructor. Kaz found neuroscience so interesting that he changed his research field completely from chemistry to biology. Since then, his major research interest has been glial biology. He has dedicated his study to the development of glial cells (oligodendrocytes and astrocytes) as well as their functions in the brain, and has become one of the top scientists in myelin biology. He is also interested in the pathophysiology of demyelinating diseases, especially multiple sclerosis. Based on his background in organic chemistry, Kaz developed a sensitive method for analyzing N-linked sugar chain structures and applied this technique to identify a new function for $\mathrm{N}$-glycans within glia. We now know that glia are more than just glue within the nervous system, and Kaz is certainly one of the key contributors who have helped to prove this.

In Prof. Mikoshiba's lab, Kaz studied proteolipid protein (PLP), a major myelin protein in the central nervous system. $\mathrm{He}$ and his colleagues generated a transgenic mouse line that contained extra copies of the PLP gene (Kagawa et al. 1994). These homozygous mice are born healthy but die within several weeks, similar to the phenotype of jimpy mice. The heterozygous mice, however, undergo severe demyelination after 5-6 months of age; given this phenotype, these mice have frequently served as a useful animal model for demyelination and for Pelizeus-Merzbacher disease caused by duplication of the PLP gene in humans. Since that time, he has been one of the top leaders in myelin research worldwide. In 1992, he established his own lab at the National Institute for Physiological Sciences. Thereafter, he has made great contributions to the Japanese glial research community by continuously obtaining huge scientific grants related to glial research and sharing these grants with other researchers, especially up-and-coming talented researchers within the field. The most recent grant he was awarded was for "Glial assembly: a new regulatory machinery of brain function and 
disorders (Ministry of Education. Culture, Sports, Science and Technology: Grant-in-Aid for Scientific Research on Innovative Areas; funded from Apr 2013 to Mar 2018)". As a result of his successful grantsmanship, glial research has now become quite popular in Japan, and the number of neuroscientists interested in myelin biology continues to grow within our society.

Kaz has made great contributions in the neuroscience field not only as a researcher but also as an officer in various scientific societies, including the International, the Asian Pacific and the Japanese Societies of Neurochemistry (ISN, APSN, and JSN, respectively). He previously held positions on the Council and as Vice President of the JSN (2005-2012), and as the President of the APSN (2006-2010). Currently he has been serving as the President of the ISN since August 2017, after serving as the Treasurer for 4 years (2013-2017). He is a great leader who has always made thoughtful and fair decisions. Kaz has also been a member of the editorial boards of numerous scientific journals: Associate Editor (2012-present) and Co-Editor-in Chief (2008-2011) of Neurochemical Research, Associate Editor (1994-present) of Developmental Neuroscience, Associate Editor (2005-present) of the Journal of Neuroscience Research, Senior Editor (2009-present) of ASN Neuro, Editorial Board (2001-present) of Glia, and Board Member (2011-present) of Brain and Behavior.

Within his home institute, he served as a Vice-Director from 2007 to 2011, and at present has been the Director of the Institute for Integrative Bioscience since 2013. Given these numerous responsibilities, he is constantly busy, yet when he is in the office, his door is always open to the members of his lab. Staff and students often come to his office to discuss their data and sometimes even to seek consultation about personal matters. Time permitting, he listens carefully and gives good advice both in and out of his office. "Nomunication" is a Japanese slang term which means communicating with people by drinking together ('nomu' means 'drink' in Japanese). This is a typical social custom in Japan, and Kaz will regularly invite people to have nomunication with him. During nomunication, he is always a source of good cheer and brings happiness to those with whom he shares this time. We all love and admire him because of his leadership in research as well as his outstanding personality.

Kaz has traveled a lot over the years. Although most of these trips have been work-related, he still manages to enjoy himself by tasting regional cuisines at local restaurants wherever he goes. So that he can order dishes for himself, he learns the local language before he visits, and now can order a meal in seven foreign languages, including English, German, French, Italian, Spanish, Korean, and Chinese, in addition to his native Japanese. Even in Japan, he likes to seek out special foods in the areas he visits. It is always fun for us to spend an uplifting time with him in nomunication.

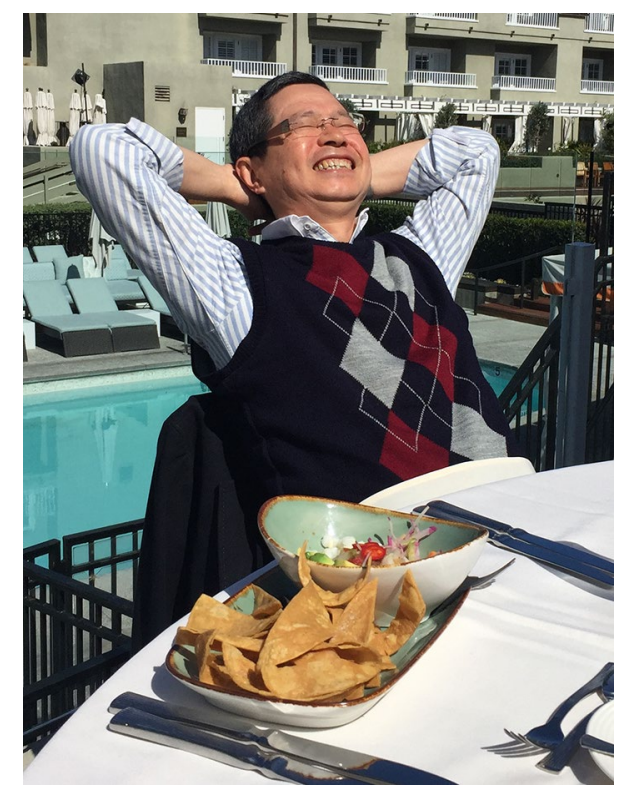

Kaz will be retiring from the institute in March of 2018. He says that he is looking forward to traveling around the Mediterranean after his retirement and vising many countries purely for fun with his charming wife Yumiko who has been a constant support behind his successes for over 39 years. However, since he will continue to be the President of the ISN until August 2019, and since many people want him to stay in research, we all wish that he would postpone this dream for just another few years. Bon voyage as you embark on this new adventure!

\section{Reference}

Kagawa T et al (1994) Glial cell degeneration and hypomyelination caused by overexpression of myelin proteolipid protein gene. Neuron 13:427-442 